\title{
Jogabilidade em jogos educacionais para crianças com discalculia
}

\author{
the playability of educational games for children with dyscalculia
}

Matheus Araujo Cezarotto e André Luiz Battaiola

A qualidade de uma interface digital é usualmente avaliada por testes de usabilidade. Nesses testes são mensurados o nível de facilidade e acessibilidade que a interface oferece para que o usuário consiga atingir os seus objetivos com eficácia, eficiência e satisfação. Todavia, no contexto dos jogos digitais educacionais para avaliar interfaces é necessário considerar também o elemento entretenimento, ou seja, a diversão. Isso posto, este estudo propõe heurísticas para a avaliação da jogabilidade em jogos educacionais voltados para crianças com discalculia do desenvolvimento. Essas crianças apresentam um déficit cognitivo que prejudica a aprendizagem na aritmética. Para tanto, utiliza-se de uma revisão bibliográfica acerca de heurísticas e recomendações para o design de jogos, bem como é realizado uma análise crítica dos estudos mapeados. Como resultado, são propostas 32 heurísticas para avaliar a jogabilidade e experiência do usuário em jogos digitais educacionais, em especial aqueles destinados para crianças com discalculia.

Palavras chave: Jogos digitais educacionais, Jogabilidade, Experiência do usuário

Specialists usually evaluate interface quality through usability tests. These tests measure the level of facility and the accessibility that the interface provides to users. In fact, the tests evaluate if users can achieve their goals with efficacy, efficient, and satisfaction. However, in the context of educational computer games, in order to evaluate a game interface specialists should consider also the entertainment (fun) aspects. Hence, this study provides a set of heuristics to evaluate the playability of educational computer games, specially those focusing on children with developmental dyscalculia. Children with dyscalculia have a cognitive deficit that affects their normal acquisition of arithmetic skills. In order to do so, the study comprises a literature review and a critical analysis of heuristics and recommendations to design games. As a result, we propose 32 heuristics to evaluate the playability of educational computer games, specially those focusing on children with dyscalculia.

Keywords: Educational computer games, Playability, User experience 


\section{Introdução}

Kucian e von Aster [2015] apontam que a sociedade ainda subestima a relevância da compreensão numérica na vida quotidiana, inclusive das crianças. Para esses autores, as habilidades matemáticas são essências no dia a dia de qualquer indivíduo. A comissão do parlamento britânico, por exemplo, classifica o conhecimento matemático como parte do conceito "capital mental", o qual aborda sobre o desenvolvimento intelectual da população e suas contribuições para a economia da sociedade [COOPER et al., 2010].

Diante disso, em uma comunidade, os transtornos de aprendizagem, como, por exemplo, a discalculia do desenvolvimento (DD), são considerados um dos principais causadores de prejuízos ao capital mental [HAASE et al., 2012]. Em síntese, a discalculia é um transtorno específico que afeta a capacidade de aprendizagem da matemática [KAUFMANN e VON ASTER, 2012]

O uso de intervenções computadorizadas para remediar a discalculia do desenvolvimento demonstra ser uma abordagem promissora [Käser et al. 2013]. Tais intervenções são denominadas como "adaptative games”, ou seja, programas de computador que por meio de algoritmo se adaptam constantemente as habilidades do usuário, ademais agregado a um ambiente de entretenimento característico dos jogos digitais, promovem o treinamento cognitivo [WILSON et al., 2006]. Portanto, esses jogos fomentam um ambiente de treinamento acessível, intenso e estimulante.

Cabe ressaltar que a acessibilidade em jogos possibilita que mesmo indivíduos com limitações possam utilizar esse tipo de mídia. Como limitações são consideradas tanto as desabilidades cognitivas (e.g. discalculia, TDAH, dislexia), quanto as desabilidades físicas (e.g. cegueira, surdez, limitações de mobilidade). Tal definição é estabelecida pelo Game Acessibility Special Interest Group (GA-SIG). Essa organização faz parte da Internacional Game Developers Association (IGDA) e vem fomentando formas de promover a acessibilidade em jogos, bem como instruindo os desenvolvedores sobre esta necessidade. [BIERRE et al., 2004]

A demanda e o uso de jogos para além do entretenimento, como na educação ou na estimulação cognitiva em uma reabilitação neuropsicológica, vem fomentando a criação e a pesquisa de jogos para essas práticas, o que é profícuo. Contudo, apesar das contribuições e dos resultados positivos com os jogos enquanto intervenções, como no caso da discalculia (e.g. Käser et al. 2013; Wilson et al., 2006), uma baixa ou nenhuma atenção tem sido atribuída aos conceitos de game design e design de interface. Esse fato limita as possibilidades de uso desses jogos, pois se desconsidera aspectos elementares como a experiência do jogador, a jogabilidade e a usabilidade [CEZAROTTO e BATTAIOLA, 2016].

Considerando isso, o objetivo deste estudo é propor um conjunto de heurísticas para analisar a jogabilidade de jogos digitais, com especial interesse para aqueles destinados às crianças com discalculia do desenvolvimento. Isso posto, a pesquisa se classifica como de natureza básica, o seu objetivo é exploratório e como método foi realizado uma revisão de literatura, bem como uma análise crítica de heurísticas e recomendações já existentes. Convém salientar que fomentar pesquisas acerca de jogos para remediar a discalculia é relevante ao considerar que, apesar da demanda, esses jogos ainda são limitados no contexto brasileiro [CEZAROTTO e BATTAIOLA, 2016].

Este estudo está organizado na seguinte estrutura: seção (2) - descrição das principais características dos jogos digitais educacionais; seção (3) - detalhamento sobre os fatores associados à aprendizagem e às intervenções, tendo como foco crianças com discalculia do desenvolvimento; seção (4) - caracterização dos aspectos norteadores para uma avaliação de usabilidade e jogabilidade; seção (5) articulação da proposta de heurísticas; seção (6) discussão dos resultados evidenciados, com especial interesse para a proposta de heurísticas. Por fim, são delineadas as considerações finais e também os direcionamentos para pesquisas futuras. 


\section{Design de jogos digitais educacionais}

Jogos digitais educacionais tem como objetivo promover a aprendizagem de algum conteúdo específico [MAYER, 2014]. Isto é, possuem um objetivo educacional claro e para atingi-lo utilizam de recursos instrucionais. Em sua estrutura, tais jogos possuem como base as características interativas e norteadoras dos jogos de entretenimento como, desafios, regras, feedback imediato e resultados quantificáveis. A configuração dessas características é a responsável por fomentar o engajamento e a emoção do jogador. [KAPP, 2012]

Tobias et al., [2014] argumentam que os jogos digitais em sua maioria são constituídos como um ambiente multimídia, já que utilizam uma combinação de imagens gráficas, texto, efeitos sonoros e comunicação oral. Consonante a isso, Rieber [2005] postula que o jogo digital educacional é um ambiente multimídia com ênfase na experiência, isto é, a aprendizagem ocorre mediante a interação do jogador com os elementos dinâmicos do jogo, o que resulta em uma experiência.
O design de jogos digitais educacionais, ao considerar as necessidades do aprendiz, envolve uma tensão no balanceamento entre os elementos de entretenimento voltados à diversão, com os elementos instrucionais, os quais se atêm a aprendizagem [MAYER, 2014]. Para tanto, inicialmente é necessário delinear informações elementares sobre o jogo a ser desenvolvido ou analisado, bem como informações sobre o seu usuário potencial.

Neste sentido, Carvalho et al., [2015] propõem um conjunto de questões que permitem descrever o cerne de um jogo educacional, ao considerar o usuário, o desenvolvedor e também os aspectos de ensino-aprendizagem (tabela 1). Acerca da análise de um jogo já existente, os referidos autores argumentam que nem sempre é possível descrever precisamente as atividades do jogo, já que ocorre uma interpretação pessoal e variável em razão do avaliador. Tal fato, não elimina a relevância das questões, pois elas permitem detectar inconsistências e contradições que possivelmente afetarão o engajamento do usuário com o jogo.

\begin{tabular}{ll} 
Sujeito & Descrição \\
\hline Quem é o jogador? & Por que o sujeito está jogando? Quais são os objetivos gerais do jogo? \\
\hline Quem é o aprendiz? & $\begin{array}{l}\text { Por que o sujeito está engajado com o jogo? Quais são os objetivos educacionais do } \\
\text { jogo? }\end{array}$ \\
\hline $\begin{array}{l}\text { Quem desenvolveu } \\
\text { o jogo? }\end{array}$ & Por que o jogo foi desenvolvido? Como o jogo está tentando transmitir o conteúdo? \\
\hline $\begin{array}{l}\text { Quem utiliza o jogo } \\
\text { para ensinar algo? }\end{array}$ & $\begin{array}{l}\text { Por que o sujeito está utilizando o jogo? Como o jogo é utilizado para ensinar algo? } \\
\text { Existem outras ferramentas sendo utilizadas em conjunto ao jogo para atingir os obje- }\end{array}$ \\
\hline
\end{tabular}

Tabela 1- Questões para descrever jogos educacionais. Fonte: Adaptado de Carvalho et al. [2015 p.174], tradução nossa

Neste contexto, a seguir são descritos aspectos do perfil cognitivo das crianças com discalculia e também aspectos do uso de jogos, enquanto intervenções neuropsicológicas.

\section{Aprendizagem e discalculia}

A discalculia do desenvolvimento (DD) é um transtorno de aprendizagem específico que afeta a capacidade de aprendizagem da matemática. Isso posto, o indivíduo com discalculia tem o seu rendimento escolar prejudicado nas habilidades matemáticas, bem como nas atividades do seu dia a dia que exigem essas habilidades [KAUFMANN e VON ASTER, 2012; SHALEV e GROSS-TSUR, 2001]. De acordo com Wilson e Dehaene [2007] a DD é causada por uma disfunção genética no funcionamento cerebral. A estimativa é que cerca de 3 a $6 \%$ das crianças em fase escolar possuem a DD, de acordo com Shalev e Gross-Tsur, 2001. No Brasil, um estudo realizado com 432 crianças de 28 escolas públicas de São Paulo, após o primeiro ciclo do ensino fundamental ( $5^{\mathrm{a}}$ ano), evidenciou que 226 crianças possuem a discalculia, o que representa uma prevalência de 7,8\% [BASTOS et al., 2016]. 
A discalculia é diagnosticada por profissionais habilitados que atuam na interface saúde e educação (e.g. médicos, psicólogos, psicopedagogos). Esses profissionais avaliam, por meio de testes padronizados, as habilidades matemáticas que estão comprometidas. Após o diagnóstico, são elaborados programas de reabilitação neuropsicológica. O objetivo da reabilitação é aprimorar a funcionalidade e qualidade de vida do indivíduo que possui a discalculia. Para tanto, é estimulada a aprendizagem da matemática por meio de técnicas cognitivas e comportamentais. [HAASE PINHEIRO-CHAGAS e ANDRADE, 2012] Na reabilitação, agregado a outras atividades, são utilizados jogos digitais educacionais para proporcionar ao indivíduo um ambiente de treinamento intenso, adaptável e motivador [KADOSH et al., 2013].

\section{Intervenções computadorizadas}

Wilson et al. [2006] listam um conjunto de vantagens cognitivas para o usuário ao utilizar jogos digitais educacionais, enquanto intervenções neuropsicológicas. Essas vantagens foram sumarizadas pelos autores do presente estudo e são listadas a seguir:

1 Jogo online.

Disponível em:

$<$ Thenumbercatcher. com>

2 Jogo online.

Disponível em:

$<$ meistercody.com $>$
- As intervenções computadorizadas possuem o potencial de manter a dificuldade das tarefas educacionais na "zona de desenvolvimento proximal - ZDP” - proposta por Vygotsky [1989]. A ZDP representa a distância entre o nível de desenvolvimento real da criança, determinado pela sua capacidade de solucionar independentemente problemas, e o seu nível de desenvolvimento potencial, ou seja, aquele determinado por meio da solução de problemas com o auxílio de uma mediação [VYGOTSKY, 1989].

- Minimizam o número de erros, uma vez que ofertam para o usuário um nível adequado de dificuldade, o que promove a estimulação cognitiva necessária para o progresso. Além do mais, alinha-se com a abordagem "aprendizagem sem erro" (do inglês Errorless learning) [MIDDLETON E SCHWARTZ, 2012], usualmente adotada em reabilitações cognitivas.

- O uso de computador (ou outras mídias digitais) permite explorar o fascínio que as crianças, adolescente e até mesmo adultos, possuem por jogos digitais. Tal fato facilita o treinamento intenso de exercícios que de outra forma seriam considerados "chatos" de realizar.

$\mathrm{Na}$ literatura se encontra diversos estudos que se atém ao desenvolvimento e a avaliação da eficácia de jogos digitais educacionais enquanto intervenções para a discalculia ou dificuldades de aprendi- zagem da matemática. Com destaque para: "Number race" Wilson et al., [2006]; "Calcularis" Käser et al., [2013]; "Number bonds" Butterworth et al., [2011]; "The Number Catcher "'; "Miester Cody"

Um dos modelos teóricos que usualmente guia o desenvolvimento de jogos enquanto intervenções para a discalculia, é o modelo do código triplo [DEHAENE e COHEN, 1995]. De acordo com Dehaene e Cohen [1995], o modelo estabelece que o processamento numérico e as operações aritméticas são realizadas por meio de três representações mentais. As representações são:

- Representação analógica associada ao senso numérico (e.g “....”)

- Representação de numerais verbais (orais e ou escritos) (e.g. "quatro")

- Representação de numerais arábicos (visuais) (e.g. “4”)

Convém destacar que para o desenvolvimento e a aprendizagem do conhecimento matemático, as três representações numéricas necessitam se relacionar [DEHAENE, 2001].

Considerando isso, Wilson et al. [2006] evidenciam a necessidade de solidificar as relações entre as representações numéricas. Para isso, os referidos autores recomendam para as intervenções computadorizadas o uso do conceito da associação repetida que simultaneamente apresenta para o usuário as três formas da representação numérica.

Já Käser et al. [2013] propõem três princípios para o design de jogos enquanto intervenções à discalculia. O primeiro deles está alinhado e se assemelha a abordagem da associação repetida descrita por Wilson et al. [2006]. O segundo princípio aborda sobre adaptabilidade e aprendizagem por andaimes (do inglês scaffolding). Ao considerar que o processo de aprendizagem das crianças é diferente, ou seja, apresenta níveis e ritmos diferenciados. Portanto, se faz necessário um ambiente de aprendizagem hierárquico que gradualmente avança com base no perfil cognitivo do usuário. Por fim, o terceiro princípio abrange o uso de diferentes tipos de conhecimento durante as atividades da intervenção. Em resumo, nesse princípio os autores estabelecem como necessário balancear a aquisição do conhecimento conceitual com a automatização. Isto é, o usuário aprende inicialmente os conhecimentos conceituais (e.g. operações aritméticas), para somente depois iniciar um treinamento para a automatização (e.g. cálculo mental).

Outro estudo que propõe contribuições para a estruturação do conteúdo instrucional em jogos focados em crianças com discalculia é a dissertação de 
Cezarotto [2016]. Esse autor evidenciou um conjunto de recomendações para o game design desses jogos. Dentre as recomendações geradas acerca do conteúdo matemático e estrutura das atividades, destaca-se:

§ O uso de módulos para ensinar os conteúdos matemáticos. Para tanto, considerar a seguinte hierarquia: Senso numérico; Contagem; Transcodificação; Adição; Subtração; Problemas Matemáticos; Multiplicação.

$\S$ Fomentar em cada módulo a automatização dos conteúdos trabalhados, para que o jogador consiga resolver os problemas no menor tempo possível, e ainda, seja capaz de aplicar os conhecimentos em contextos diferenciados.

$\S$ As atividades de cada módulo de conteúdo, precisam ser estruturadas de forma adaptável ao perfil cognitivo do usuário.

§ É necessária a prática repetida das atividades. Contudo, em sua aplicação considerar estratégias para que a repetição excessiva não prejudique a motivação do usuário.

$\S$ Jogos configurados como monousuário e caracterizados por atividades individuais são mais eficazes para crianças com discalculia.

§ Para aplicação da intervenção computadorizada é recomendado o uso de mediação direta por profissionais qualificados (e.g. pedagogos, psicólogos, psicopedagogos)

Contudo, apesar das contribuições e dos resultados positivos dos estudos acima mencionados, uma baixa ou nenhuma atenção é estimulada nas pesquisas para os conceitos de game design ou de design de interfaces. Uma vez que tais pesquisas em sua maioria são provenientes da área da computação ou da neuropsicologia, assim apresentam um interesse distinto. Tal despreocupação se caracteriza como uma lacuna e acaba por limitar as possibilidades de uso desses jogos, pois são desconsiderados aspectos elementares do design de jogos e design de interfaces, a citar a experiência do jogador, jogabilidade, usabilidade etc. [CEZAROTTO e BATTAIOLA, 2016].

\section{Usabilidade e experiência do usuário em jogos digitais educacionais}

Gomes e Padovani [2005] argumentam que a qualidade de um software educativo está associada à capacidade que esse artefato possui para responder a requisitos e necessidades do usuário associadas à aprendizagem. Segundo os referidos autores, a no- ção de qualidade é elementar para estabelecer níveis de facilidade e acessibilidade aos usuários em relação às funções do sistema. Dessa conjuntura, destaca-se a noção de usabilidade como fundamental para promover a qualidade do sistema.

A usabilidade é o nível com que um sistema, produto ou serviço é capaz de ser utilizado por usuários específicos para atingir seus objetivos com eficácia, eficiência e satisfação em um contexto de uso [ISO 9241-210]. De acordo com a referida norma ISO, a eficácia diz respeito à precisão e a completude com que o usuário atinge os objetivos. A eficiência representa os recursos despendidos em relação a precisão e completude com que o usuário atinge os objetivos. A satisfação é definida como a ausência de desconfortos, isto é, representa atitudes positivas no uso do sistema, produto ou serviço.

Entretanto, para Federoff [2002] no contexto dos jogos digitais em razão da existência da jogabilidade (playability), a qual está integrada à usabilidade, isso implica novos elementos para a mensuração da qualidade do sistema. Consonante a isso, Pagulayan et al., [2003] ressaltam que a qualidade do jogo digital depende também do fator entretenimento, isto é, a diversão e emoção que o artefato é capaz de estimular no jogador. Portanto, argumenta-se que é necessário que o jogo digital educacional viabilize que o jogador alcance uma determinada produtividade em termos de aprendizagem, contudo, esse jogo necessita também promover entretenimento (diversão). Note-se que o fator entretenimento está associado à experiência do usuário.

Preece et al., (2005) evidenciam a relevância em considerar a experiência do usuário, ou seja, como o usuário se sentirá ao interagir com o sistema. Para essas autoras, enquanto as metas de usabilidade se atém aos critérios específicos de usabilidade (e.g. eficiência), por sua vez, as metas da experiência do usuário se atém em explicar a qualidade da experiência (e.g. ser divertido). Ou seja, a experiência do usuário está além de apenas possibilitar a realização da tarefa, busca desenvolver sistemas interativos agradáveis, divertidos, esteticamente apreciáveis, etc. [PREECE et al., 2005]

Quando um jogo não é agradável ou divertido, quando é cansativo, chato, demasiadamente difícil ou fácil, o usuário se ressente do entretenimento. Esse problema afetará a usabilidade do sistema, dado que, nos jogos digitais, apesar da usabilidade e da diversão estarem alocados em categorias diferentes, não é possível separá-las, uma vez que uma influencia a outra. [BARENDREGT, 2006]

Essa influência é didaticamente evidenciada por Barendregt [2006 p.8], ao propor uma hierarquia para as necessidades do jogador em jogos digitais (figura 1). A proposta tem como base o estudo de Jordan [1999 p. 209] que define três níveis de hie- 
rarquia para as necessidades do usuário, ao considerar a conhecida hierarquia das necessidades humanas definida por Maslow [1970].

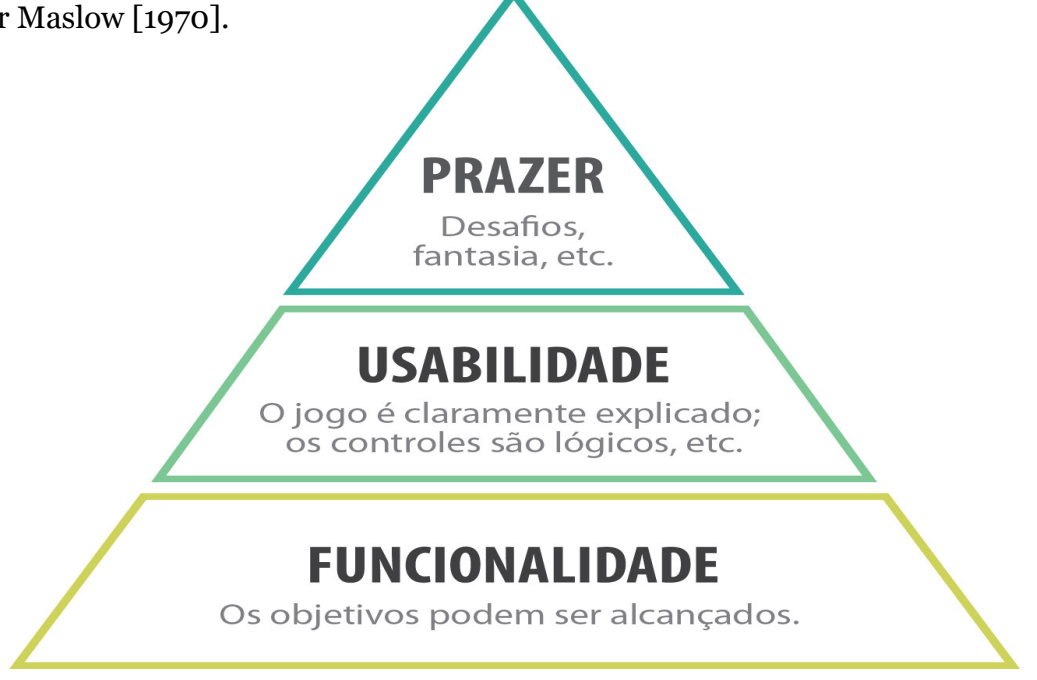

Figura 1- hierarquia de necessidades do jogador. Fonte: Adaptado de Barendregt [2006], tradução nossa.

Em síntese, a hierarquia de Jordan [1999] estabelece que quando o usuário atinge as suas necessidades mais baixas da hierarquia, ele automaticamente inicia a busca pelas necessidades mais altas da escala. Porém, o referido autor salienta que mesmo quando as necessidades mais básicas são atingidas, os usuários ainda se frustrarão se os objetivos mais altos da hierarquia não forem alcançados [JORDAN, 1999].

Ao aplicar essa hierarquia para os jogos digitais, Barendregt [2006] explica que no nível inicial denominado funcionalidade, os objetivos estipulados pelo jogo precisam ser funcionais ao ponto de serem possivelmente alcançados pelo jogador. No nível usabilidade, as maneiras de jogar precisam ser claras e os controles não devem promover desconfortos para o jogador. Por fim, no nível prazer o jogo precisa ser divertido, ao fornecer desafios interessantes e fantasias que sejam significativas para o jogador.

Assim sendo, argumenta-se que essa pirâmide de necessidades pode ser utilizada para mensurar a qualidade dos jogos digitais educacionais, uma vez que para atingir um nível de qualidade em aspectos de aprendizagem, o jogo educacional precisará contemplar todos os níveis da hierarquia. Ou seja, o objetivo central do jogo educacional é fomentar a aprendizagem, para isto ele precisa ser funcional, fácil de utilizar e ainda promover uma experiência prazerosa para o usuário. Para tanto, de modo a clarificar essa relação entre aprendizagem, hierarquia de necessidades do jogador e estrutura dos jo- gos digitais se faz necessário caracterizar dois conceitos, o gameplay e a jogabilidade (playability). Aguiar e Battaiola [2016], por meio de uma análise detalhada na literatura, propõem uma definição consensual para os termos mencionados.

Em suma, o gameplay é o que promove o fluxo do jogo e estabelece a interação com as mecânicas, logo resultando em jogabilidade. Assim, o gameplay abrange o fluxo de jogo, interação, regras e mecânicas. Já o termo jogabilidade está associado à experiência do jogador, portanto, abrange a satisfação do usuário, a facilidade de uso e a capacidade de motivar o jogador. [AGUIAR e BATTAIOLA, 2016]

Considerando a discussão levantada elaborou-se o diagrama 1, o qual representa uma proposta de mapeamento das relações entre os conceitos discutidos nesta pesquisa: gameplay, jogabilidade e hierarquia de necessidades do jogador.

Por meio das relações do diagrama 1, são delineadas algumas inferências que contribuem para a análise da jogabilidade e experiência do usuário em jogos digitais educacionais. As inferências são descritas a seguir.

Inicialmente é evidenciado no diagrama a relevância em delinear o perfil cognitivo do usuário do jogo, ou seja, suas necessidades e requisitos relacionados à aprendizagem. 
Ao começar a interagir com o jogo digital educacional, o usuário se depara com a funcionalidade do sistema que representa o primeiro nível da escala.
Logo, o usuário precisa identificar e compreender os objetivos do jogo, bem como por meio da interface ser capaz de atingir tais objetivos.

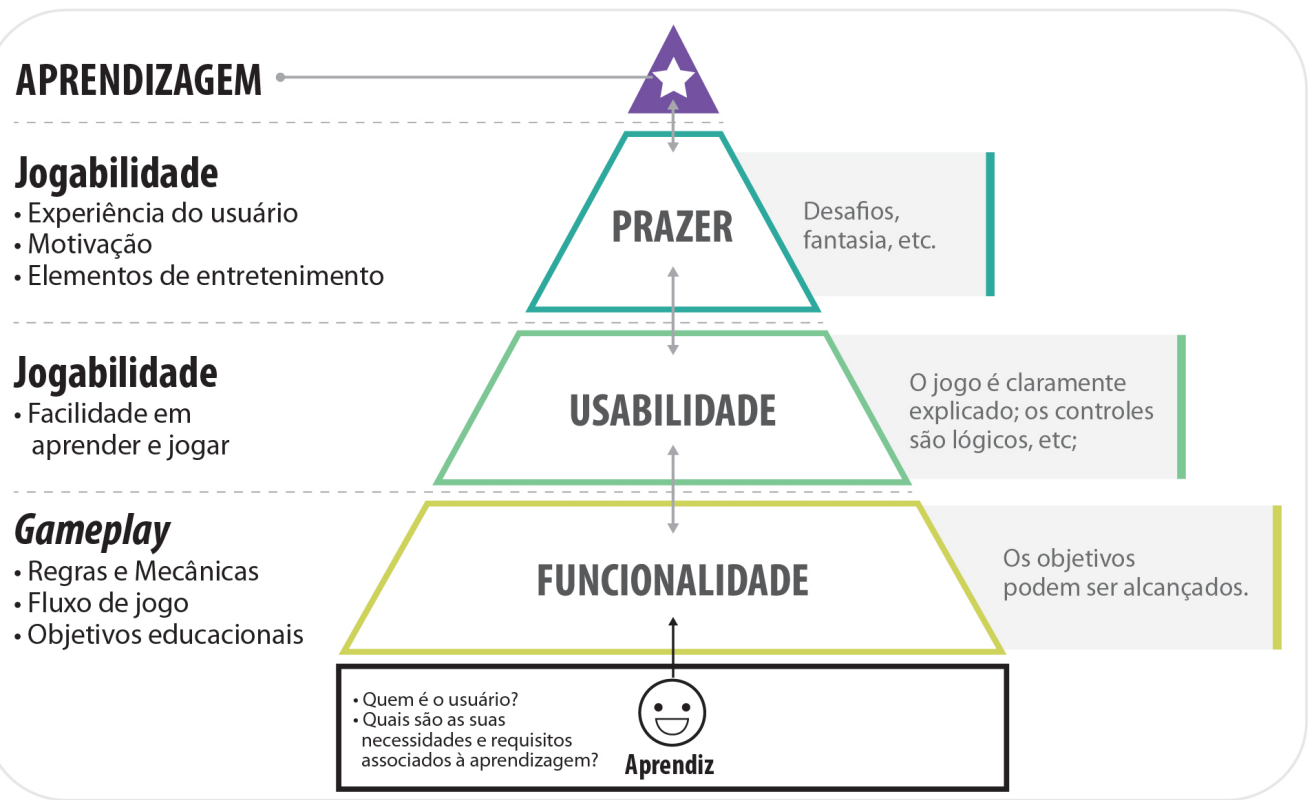

Diagrama 1- a relação nos jogos digitais educacionais entre gameplay, jogabilidade e necessidades do jogador. Fonte: Os autores com base em Jordan [1999]; Barendregt [2006]; Aguiar e Battaiola [2016]

Convém salientar que os objetivos de jogo estão associados com os objetivos educacionais planejados para o artefato. Portanto, esse nível da hierarquia representa o gameplay do jogo, em que o jogador ao interagir com o sistema se depara com o fluxo de jogo por meio das mecânicas e regras.

No segundo nível da escala, o jogador começa a observar a usabilidade do jogo, ou seja, ele percebe se os objetivos e regras são claramente explicados e também se os controles são lógicos. Assim, representa uma parte da jogabilidade, o que compreende o nível da facilidade que é para o usuário aprender e jogar.

Em seguida, ainda associado a jogabilidade, o usuário avança para elementos mais específicos e subjetivos associados à experiência de jogo, isto é, como ele se sente ao interagir com o sistema. Neste nível se observa aspectos motivacionais, elementos de entretenimento, ademais a capacidade de fomentar emoções ou prazer ao jogador.

Por fim, no topo da pirâmide alocou-se a aprendizagem. Pois, argumenta-se que um jogo digital educacional de qualidade necessita elementarmente fomentar para o usuário funcionalidade, usabilidade e prazer, para somente então, ser capaz de promover situações de ensino-aprendizagem.
Isso posto, buscou-se na literatura pesquisas que apresentam heurísticas para a mensuração da qualidade de jogos, ao se considerar o gameplay e a jogabilidade

\section{Heurísticas para jogos educacionais}

Em síntese, a avaliação heurística com especialistas consiste em uma mensuração da qualidade de um software com base em um conjunto de princípios (heurísticas). O objetivo é inspecionar a interface em termos de sua facilidade de uso. [GOMES e PADOVANI, 2005] Existem diversos autores que definem heurísticas para a avaliação de interfaces, a citar por exemplo Bastien e Scapin [1993], Nilsen [1995], entre outros. Os quais, apesar de suas contribuições e possível aplicação em jogos, carecem de uma especificidade que esse artefato exige. Portanto, por meio de uma revisão da literatura foram selecionados três estudos que propõem heurísticas ou recomendações voltadas para jogos digitais: Federoff [2002]; Desurvire e Wiberg [2009]; Cezarotto e Battaiola [2016]. $\mathrm{O}$ intuito foi mapear, analisar e sintetizar um conjunto de heurísticas que permitissem mensurar a qualidade do gameplay e da jogabilidade em jogos 
digitais educacionais, em especial aqueles destinados às crianças com discalculia.

Federoff [2002] por meio de revisão na literatura mapeou um conjunto de heurísticas voltadas para o game design, além disso realizou avaliações com especialistas da área de jogos em um estudo de caso, o que fez emergir novas heurísticas. No total, a autora estruturou um conjunto de 40 heurísticas.

Desurvire e Wiberg [2009], também com base em uma revisão de literatura, propõem a $\mathrm{HEP}$ - Heuristics for Evaluating Playability. Convém ressaltar que a pesquisa de Federoff [2002] faz parte dessa revisão. As heurísticas (50 no total) foram coletadas e refinadas especificamente para o contexto dos jogos.

Já Cezarotto e Battaiola [2016], por meio de uma revisão de literatura, estudo de caso com usuários e avaliação com especialistas da área de jogos e da psicologia, propõem um conjunto de recomendações (20 no total) para fomentar a motivação em jogos digitais destinadas as crianças com discalculia.
Para análise, as heurísticas e recomendações foram classificadas pelos pesquisadores deste estudo, tomando-se por bases as suas características, em quatro categorias:

$\S$ gameplay - aspectos associados à aprendizagem de crianças com discalculia;

§ gameplay nível funcionalidade mecânicas, regras e fluxo de jogo;

$\S$ jogabilidade nível usabilidade- facilidade de uso e elementos de interface;

$\S$ jogabilidade nível prazer - experiência do usuário.

A proposta de heurísticas deste estudo está organizada na tabela 2.

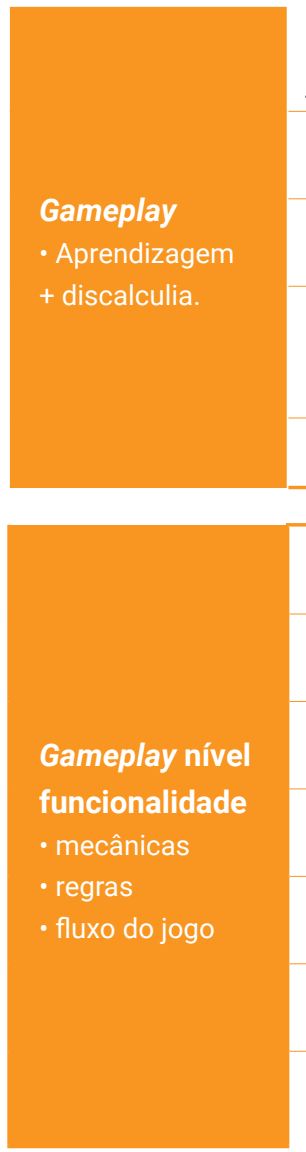

As atividades matemáticas seguem um conteúdo hierárquico que gradualmente exige mais do jogador, ao considerar as habilidades do jogador (Scaffolding) [4]

As atividades de jogo estimulam primeiro os conhecimentos conceituais para depois treinar a automatização [4;5]

As atividades de jogo utilizam o conceito da associação repetida (uso das representações numéricas: analógica; verbal; arábica) [2;4]

As atividades de jogo estimulam a prática por meio da repetição. Contudo, o jogo considera estratégias para não prejudicar a motivação do usuário em razão da repetição excessiva. [5]

O jogo é configurado como monousuário, caracterizado por atividades individuais [5]

O jogo realiza um nivelamento inicial para posicionar o jogador em desafios adequados com base nas suas habilidades. [6]

Antes de começar, o sistema apresenta para o jogador instruções claras sobre os objetivos e as regras de jogo. $[1 ; 3 ; 6]$

O jogo permite a tutoria, ao possibilitar a mensuração de dados do usuário em uma interface para o profissional mediador. [6]

O jogo é justo, as atividades favorecem as habilidades do jogador em relação à sorte. $[1 ; 5]$

O jogador não é penalizado com feedback negativo por erros cometidos. [3;6]

O jogador recebe ajuda (dicas para o êxito na tarefa) quando tem problemas para avançar. [3;6]

Os erros cometidos são possíveis de recuperação para não impedir o avanço do jogador. [3;6] 
Os controles de jogo são intuitivos e interpretados de forma natural pelo jogador. [1;3]

A interface é o menos intrusiva possível (na plataforma PC, recomenda-se ocultar a interface principal durante o jogo). [1]

O jogador consegue facilmente identificar a sua pontuação/status no jogo (porém tais elementos não interferem no jogo). [1;3]

Existem meios para a prevenção e a recuperação de erros (e.g. mensagens de alerta). [1]

O jogador não precisa ler um manual para jogar. [3]

Jogabilidade

nível usabilidade

- facilidade de uso

- elementos

de interface

O layout da tela de jogo é eficiente, integrado e visualmente agradável. [1;3;6]

A interface é consistente no uso da tipografia e na cor, o que promove a legibilidade. Apresenta ainda, consistência na organização, agrupamento e localização dos elementos. [1;3;6]

A interface possui elementos gráficos que permitam a navegação no sistema de jogo. Assim, oferece os controles básicos de menu (iniciar, pausar, salvar, voltar, ajustes e ajuda). [1;3;6]

O sistema permite pausar e salvar em diferentes estados do jogo para retornar depois. [1;3]

A interface de jogo possui elementos gráficos e sonoros que promovem o feedback imediato para as ações do jogador $[1 ; 6]$

A interface de jogo possui elementos gráficos que promovem a autoeficácia ao mostrar o desempenho e o progresso do jogador (e.g. pontos; insígnias; quadro de desempenho; missões; coleção de recursos; progresso; níveis.) [6]

O jogo promove feedback positivo com diferentes níveis de recompensas para as ações corretas do jogador. [3;6]

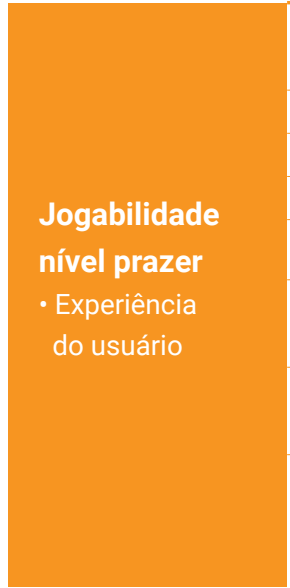

O ritmo de jogo instiga o jogador, em um nível intermediário, sem causar frustrações (desafios nem muito grandiosos nem muito insignificantes) [1;3;6]

O fluxo de jogo é balanceado, ou seja, não existe uma única e definitiva maneira de vencer. [1;3] São oportunizadas chances para que o jogador sobreviva no jogo (e.g. vidas, tentativas). [3;6] O jogador tem um senso de controle e influência no mundo de jogo (consegue fazer escolhas). [3] O jogo utiliza do elemento humor (e.g. personagens carismáticas e engraçados). [3]

Uso de personagem como estímulo visual para o jogador, atraindo a sua atenção para a atividade. [6]

Uso de cenários como parte do contexto lúdico de jogo. A expressão gráfica promove um apelo emocional, despertando a atenção e curiosidade do jogador. [6]

Uso de narrativa como base para o contexto lúdico e ficcional de jogo. Permite atuar como uma referência na construção dos elementos de jogo como, personagens, cenários, mecânicas, atividades e recompensas. [6]

Tabela 2- Heurísticas para avaliação da jogabilidade e do gameplay em jogos digitais educacionais. Fonte: Os autores com base em: [1] Federoff [2002]; [2] Wilson et al. [2006]; [3] Desurvire e Weber [2009]; [4] Käser et al. [2013]; [5] Cezarotto [2016]; [6] Cezarotto e Battaiola [2016]

\section{Heurísticas no processo de game design}

Para fomentar o uso das heurísticas evidenciadas neste estudo, argumenta-se como necessário que elas sejam contextualizadas e analisadas tendo como base o processo de game design de um jogo educacional. Ou seja, como e quando as heurísticas podem ser aplicadas no desenvolvimento do jogo educacional.

Isso posto, na literatura, em razão dos avanços tecnológicos e o uso cada vez mais frequente de jogos digitais nas práticas pedagógicas, existe uma preocupação e discussão crescente entre pesquisadores 
e desenvolvedores, sobre como estimular uma qualidade mínima para o jogo educacional. Assim, pesquisas são estimuladas para compreender o processo de desenvolvimento desses jogos, tais pesquisas abrangem os mais variados enfoques, por exemplo, ferramentas necessárias, elementos de jogo, stakeholders envolvidos e etapas de concepção. A citar as pesquisas de Carvalho et al., [2015]; Cezarotto [2016], entre outras.

Dessa forma, ressalta-se que no âmbito do design de jogos educacionais não existe um único modelo, processo, ou ainda, fórmulas de sucesso a se considerar para fomentar o êxito no design de jogos educacionais. Isto é, desenvolver um jogo que seja divertido e que estimule a aprendizagem do conteúdo. Pelo contrário, existem diversas abordagens para a concepção dos jogos educacionais. Contudo, na literatura é possível observar que determinadas abordagens recebem destaque, de acordo com Cezarotto [2016].
Assim, ao considerar o escopo deste estudo, foram selecionadas duas abordagens. Essas abordagens, além de serem amplamente referenciadas na literatura, são complementares e permitem incorporar as heurísticas aqui evidenciadas de uma forma pragmática e flexível, na prática do design de jogos educacionais. As abordagens são fomentadas por Chandler [2012] e Winn [2008].

A proposta de Chandler [2012] foi selecionada pois se caracteriza como um framework básico, o qual contempla o processo de desenvolvimento de um jogo digital, desde o conceito à pós-produção. A autora denomina a proposta como o ciclo básico de produção para jogos digitais. Esse ciclo está estruturado em quatro fases principais, as quais estão organizadas no diagrama 2 e descritas em síntese a seguir. Convém salientar que apesar da proposta de Chandler [2012] não ser específica para jogos educacionais, ela é aplicável ao contexto educacional. Em razão da amplitude e elementaridade de suas fases, o que abrange de forma básica e estrutural a diversos processos de desenvolvimento de jogos.

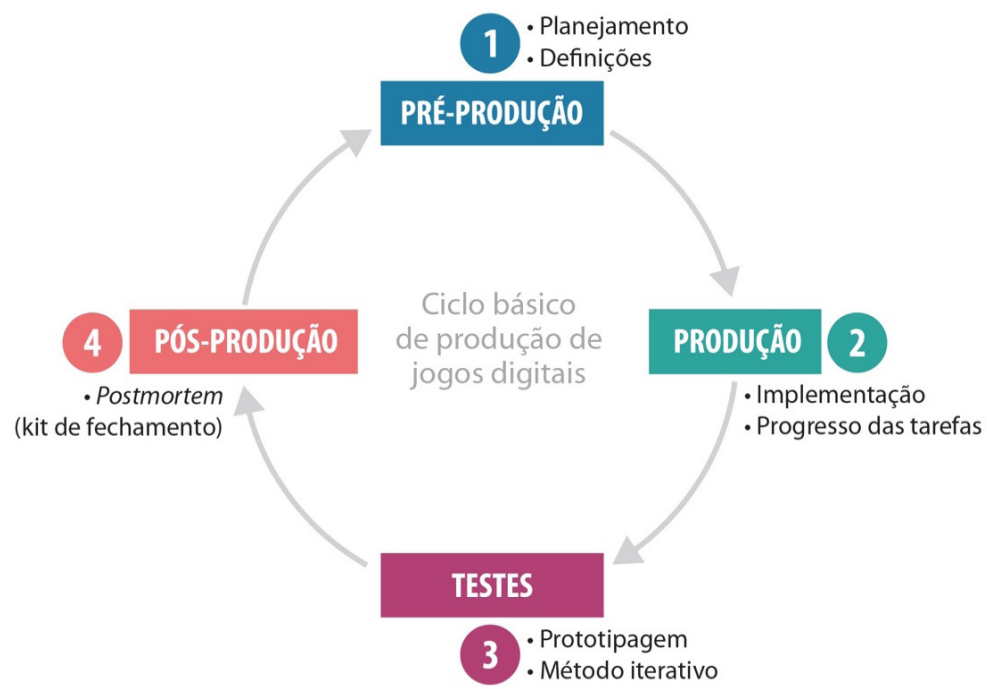

Diagrama 2: Ciclo básico de produção para jogos digitais. Fonte: Os autores com base em Chandler [2012]

Na pré-produção é iniciado o desenvolvimento do jogo, para tanto são definidos os conceitos mais básicos e norteadores do projeto. Segundo Chandler [2012] o objetivo é criar um planejamento, que guiará a equipe até o final do desenvolvimento do jogo. Assim, a pré-produção abrange além de informações sobre o conceito e o planejamento do jogo, as suas caraterísticas, limitações e exigências projetuais. Essas informações, são registradas em documentos técnicos do projeto como, o high con- cept e o game design document (GDD) [CHANDLER, 2012]. Argumenta-se que é neste momento que são delineadas as preferências e necessidades do público-alvo do jogo, inclusive as associadas à aprendizagem. Além do mais, são evidenciadas as demandas e necessidades do mediador do jogo educacional. Portanto, tais informações coletadas na fase de pré-produção, representam a categoria gameplay e jogabilidade (nível prazer) da proposta de heurística (tabela 2). Essas categorias são flexí- 
veis e necessitam de customizações, em razão do público-alvo e conteúdo a ser ensinado com o jogo educacional.

Em seguida na fase produção, as informações previamente planejadas são colocadas em prática, isto é, inicia-se realmente a produzir o jogo. Nesta fase, as características do projeto podem ser alteradas, adicionadas, ou até mesmo removidas da documentação já elaborada. Em suma, esta fase tem seu foco na criação da programação do jogo (códigos), bem como um acompanhamento do progresso do desenvolvimento do jogo (ferramentas, recursos e implementação), somado a finalização das tarefas estipuladas no cronograma inicial. [CHANDLER, 2012] Considerando isso, as heurísticas propostas neste estudo não se aplicam nesta fase.

A próxima fase do ciclo de produção proposto por Chandler [2012] é denominada testes (prototipação). Essa fase é considerada como uma das mais relevantes no processo de game design, pois é nela que o jogo é verificado para assegurar o seu funcionamento correto, ao corrigir possíveis erros (bugs) na sua programação, bem como alinhar o jogo com as necessidades e preferências do jogador [CHANDLER, 2012]. No contexto dos jogos educacionais, nesta fase são necessários também testes para a eficácia do jogo, o que abrange a aprendizagem fomentada e a motivação do usuário, como recomenda Mattar [2010]. Assim, as heurísticas, propostas neste estudo, em totalidade se aplicam nesta fase como uma avaliação prévia realizada pelo desenvolvedor, a qual permite evidenciar possíveis problemas no jogo educacional. Para posteriormente, ainda na fase de testes, a equipe inspecionar o jogo com o usuário-aprendiz e realizar possíveis alterações.

Por fim, na fase de pós-produção (finalização), após o jogo ter a aprovação final em todos os testes é realizado o encerramento do projeto, o que finaliza oficialmente a produção do jogo. Desse modo, a equipe prepara o denominado postmortem (kit de fechamento ou memorial do projeto) contendo uma revisão dos pontos positivos e negativos observados durante o desenvolvimento do jogo, ademais, o que a equipe aprendeu ao longo do projeto. Essas informações são destinadas a projetos futuros ou também para possíveis atualizações no jogo, visto que este memorial contém todas as informações e arquivos utilizados para a criação do jogo (e.g. documentação de design, arquivos de áudio, arquivos de arte) [CHANDLER, 2012].

Convém salientar que em um projeto, usualmente são realizados mais de um ciclo de produção, segundo Chandler [2012]. Tal fato se justifica uma vez que a indústria de jogos em geral utiliza em seu processo de desenvolvimento o modelo iterativo de produção de software [SCHELL, 2010].

Após esta discussão, no diagrama 3 é graficamente organizada a interação entre o ciclo de produção [CHANDLER, 2012] e as heurísticas geradas neste estudo (tabela 2).

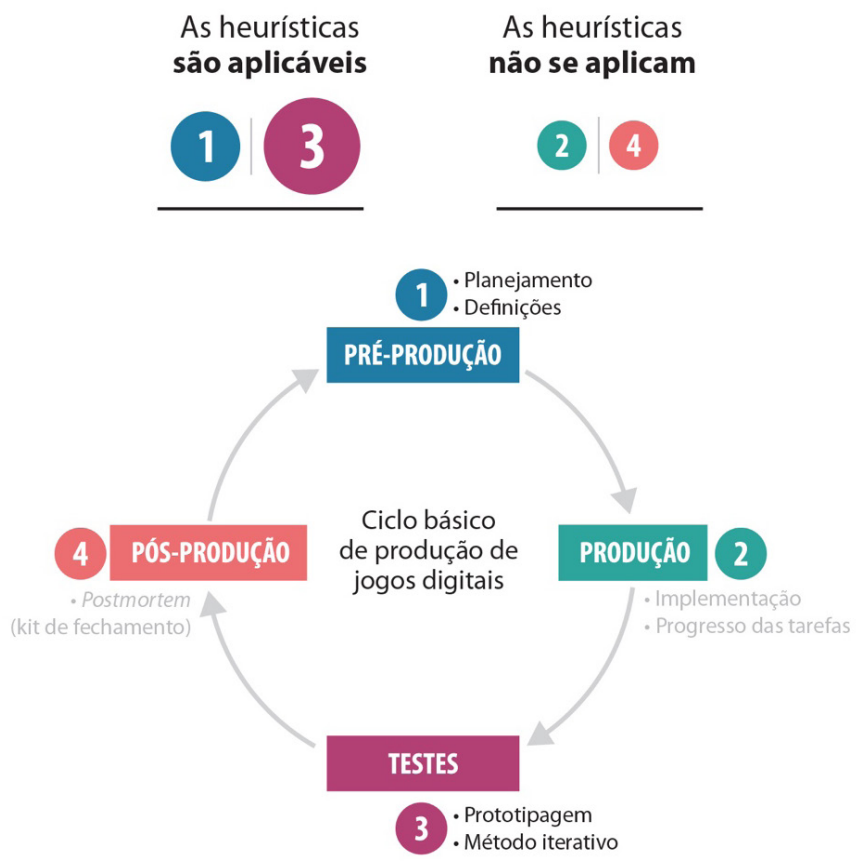

Diagrama 3: A relação entre o ciclo básico de produção para jogos digitais e a heurísticas geradas neste estudo. Fonte: Os autores com base em Chandler [2012] 
Após delinear uma interação prática entre as heurísticas evidenciadas neste estudo e o ciclo básico de produção de jogos digitais [CHANDLER, 2012], argumenta-se como complementar realizar uma discussão mais conceitual, ao considerar as heurísticas pelo viés do framework DPE (do inglês design, play, and experience). O framework (diagrama 4) foi proposto por Winn [2008] e se caracteriza como uma expansão do popular modelo MDA (do inglês Mechanics, Dynamics and Aesthetcis) [HUNICKE, LEBLANC e ZUBEK, 2004], ao concentrar o seu interesse para jogos educacionais. A ideia central da proposta está na descrição da relação entre o designer e o jogador, o que fomenta a construção da experiência de jogo. Em síntese, para cada camada do modelo (Design; Jogar; Experiência - tradução nossa) são enunciados quatro subcomponentes do design de jogos educacionais, os quais são sucintamente descritos a seguir, com base em Winn [2008].

No subcomponente aprendizagem, é desenvolvido pela equipe o conteúdo e a abordagem pedagógica para promover a aprendizagem de determinado conteúdo, por meio da interação com o jogo. Em síntese, o objetivo central é definir no início do processo de design os objetivos de aprendizagem. Ao considerar que tais objetivos constituem não somente a base do jogo educacional, mas permitem posteriormente a avaliação da aprendizagem do jogador.

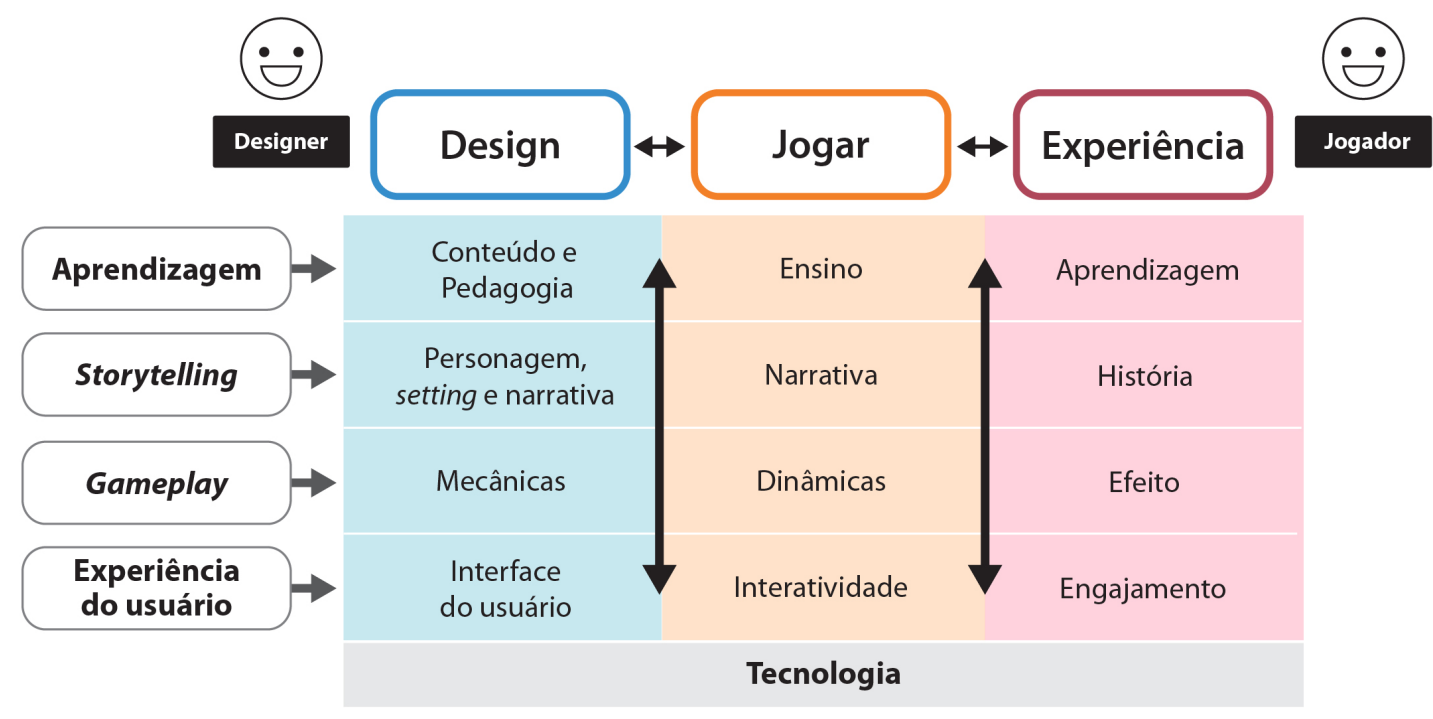

Diagrama 4: framework DPE. Fonte: os autores com base em Winn [2008 p.1015], tradução nossa.

Em seguida, no subcomponente storytelling, são consideradas duas histórias, uma pela perspectiva do designer e a outra pela perspectiva do jogador. Inicialmente, o designer idealiza o storytelling para promover o engajamento e estimular o conteúdo. Para tanto, são definidos personagens, narrativa, ambientação e cenários. Posteriormente, durante o ato de jogar, acontece uma combinação do storytelling estipulado pelo designer com a escolhas e interação do jogador, o que reflete na construção da história do jogador na camada experiência.

Já o subcomponente gameplay, é definido no framework como o que jogador pode fazer no jogo, o que abrange desde as suas escolhas, estratégias e também contempla a experiência de jogo. Inicialmente, na camada design são definidas pela equipe de desenvolvimento as mecânicas, que abrangem as regras, os desafios e os objetivos do jogo. As mecânicas no framework DPE se alinham com a categoria gameplay (nível funcionalidade) das heurísticas evidenciadas no presente estudo. Em seguida, na camada jogar, as dinâmicas são o resultado comportamental do jogador para as regras estipuladas, isto é, as estratégias desenvolvidas pelo jogador para superar os desafios e objetivos de jogo. Por fim, na última camada (experiência), o termo efeito representa as experiências e emoções do jogador. Esse efeito se alinha com a categoria jogabilidade (nível prazer) proposta neste estudo (tabela 2). Neste sentido, Winn [2008] salienta que a equipe desenvolvimento apenas tem controle sobre a primeira camada (design), já que mesmo idealizando a experiência de jogo, essa só poderá ser confirmada ou aprimorada mediante um ciclo iterativo de 
testes com o usuário. Uma vez que para o referido autor, o ato de jogar é uma experiência mediada e, portanto, é influenciado não apenas pelo seu design, mas igualmente pelo jogador. Assim, no projeto do jogo educacional é essencial ponderar sobre os aspectos cognitivos, sociais e culturais do jogador-aprendiz.

O subcomponente experiência do usuário (UX), é caracterizado no framework como o componente mais visível para o jogador, uma vez que se atém principalmente a interface de jogo. Assim, o propósito da interface com o jogador é criar um veículo que permita a realização dos resultados de aprendizagem planejados. Para o Winn [2008], o game design se manifesta por meio da interface, já que ela abrange tudo o que o jogador vê, escuta, interage, bem como é o que permite que a interação ocorra (e.g. sistema de controle). Note-se que no framework DPE o subcomponente experiência do usuário está atrelado principalmente a interface de jogo e as suas possibilidades de interação. Dessa forma, não abrange o prazer e emoção que o jogo promoverá ao jogador, sendo tais fatores contemplados no subcomponente do framework - gameplay (efeito), o qual foi descrito anteriormente.

O framework DPE possui ainda uma camada de base denominada tecnologia, que representa a plataforma em que o jogo está sendo desenvolvido. Ou seja, abrange informações como as capacidades, limitações e recursos tecnológicos necessários para implementar o jogo.

Após esta breve apresentação do framework DPE [WINN, 2008], observa-se que a proposta deheurísticas gerada neste estudo pode ser utiliza em conjunto à sua camada "design" (diagrama 4), a qual está mais próxima do ponto de vista da equipe de desenvolvimento. Todavia, como já mencionado, as heurísticas precisam ser customizadas em razão do público e conteúdo do jogo, em especial na categoria gameplay (aprendizagem/conteúdo) (tabela 2). Assim, na tabela 3 é estruturada a relação entre as heurísticas deste estudo e o framework DPE [WINN, 2008].

\section{Discussão}

As heurísticas delineadas nesta pesquisa permitem a inspeção da jogabilidade e da usabilidade em jogos digitais educacionais, em especial para aqueles utilizados como intervenções a discalculia. Tal avaliação por meio das heurísticas pode ser adotada como a prévia de uma análise posterior e mais detalhada com o usuário do jogo. Ou seja, é nítido para os pesquisadores desta pesquisa que não é possível avaliar por completo se o jogo é fácil de utilizar e se ele fomenta experiências positivas sem a participação do usuário.

Contudo, é possível verificar se os elementos e as características necessárias para fomentar a jogabilidade estão sendo contemplados no jogo analisado, o que auxilia na construção de futuras pesquisas com o usuário. Esse fato foi discutido, ao contextualizar as heurísticas com o ciclo básico de produção de jogos digitais [CHANDLER, 2012] e o framework DPE [WINN, 2008]. Além do mais, a proposta se estrutura em dois conceitos norteadores dos jogos digitais educacionais, a jogabilidade e o gameplay. Ao alocar esses conceitos na pirâmide hierárquica das necessidades do jogador [JORDAN, 1999; BARENDREGT, 2006], foi possível inferir que as situações de ensino aprendizagem só poderão ser estimuladas e propiciadas para o usuário se o sistema de jogo fomentar funcionalidade (objetivos claros e alcançáveis), usabilidade (facilidade em aprender e jogar) e por fim, mas não menos importante, estimular o prazer (diversão e entretenimento).

\begin{tabular}{l|ll}
$\begin{array}{l}\text { Subcomponente (framework DPE) } \\
\text { Winn (2008) }\end{array}$ & $\begin{array}{l}\text { Camada Design (framework DPE) } \\
\text { Winn (2008) }\end{array}$ & $\begin{array}{l}\text { Categoria de heurísticas } \\
\text { Evidenciadas no presente estudo }\end{array}$ \\
\hline Aprendizagem & Conteúdo & $\begin{array}{l}\text { Gameplay (Nível Funcionalidade) } \\
\text { Conteúdo, Objetivos e Regras }\end{array}$ \\
\hline Storytelling & Personagem, narrativa, setting & $\begin{array}{l}\text { Jogabilidade (Nível Prazer) } \\
\text { Elementos de Entretenimento }\end{array}$ \\
\hline Gameplay & & Gameplay (Nível Funcionalidade) \\
\hline Experiência do usuário & Mecânicas & Objetivos e Regras de jogo \\
\hline
\end{tabular}

Tabela 3: Relação entre as heurísticas geradas no estudo e o modelo DPE [Winn, 2008]. Fonte: Os autores 
Dessa forma, alinha-se com a necessidade ressaltada por Preece et al. [2005] de não apenas considerar a usabilidade do artefato, mas avançar e contemplar também a experiência do usuário. Assim, considera-se insuficiente que o jogo educacional apenas funcione e seja fácil de interagir, pois ele precisa também ser divertido, atrativo e motivador para o usuário [PAGULAYAN et al., 2003]

Para tanto, é essencial conhecer em detalhes o conteúdo, os objetivos pedagógicos, bem como o perfil cognitivo e as preferências do usuário [CARVALHO et al., 2015]. No contexto de jogos para crianças com discalculia já existem estudos ressaltando as necessidades cognitivas desse usuário [KÄSER et al., 2013; KADOSH et al., 2013], todavia, ainda são limitadas as pesquisas acerca da experiência desse usuário, ou seja, como ele se sente ao interagir com jogos em uma reabilitação neuropsicológica.

\section{Considerações finais}

O presente estudo, por meio de uma discussão teórica sobre a jogabilidade em jogos digitais educacionais, traz contribuições para potencializar a qualidade de jogos já existentes ou em produção, para crianças com discalculia do desenvolvimento.

A principal contribuição da pesquisa são as 32 heurísticas estruturadas nas categorias gameplay e jogabilidade, que atendem ao objetivo inicialmente proposto. Tais heurísticas foram mapeadas, analisadas e formuladas pelos pesquisadores, tomando-se por base principalmente os estudos de Federoff
[2002]; Desurvire e Wiberg [2009]; Cezarotto e Battaiola [2016], entre outros autores. Também foram evidenciados, por meio da articulação entre autores do referencial teórico (e.g. Jordan 1999; Barendregt, 2006; Aguiar e Battaiola, 2016), relações entre os conceitos de jogabilidade e experiência do usuário no âmbito dos jogos digitais educacionais. Essa relação gerou a construção de uma representação gráfica de síntese (diagrama 1), a qual permite evidenciar o percurso e os requisitos para fomentar situações de aprendizagem nesse artefato.

Além disso, ao contextualizar as heurísticas com o processo de game design, por meio dos estudos de Chandler [2012] e Winn [2008], buscou-se delinear de forma pragmática e flexível, como e quando as heurísticas podem ser aplicadas na produção do jogo educacional.

Contudo, apesar das contribuições geradas, este estudo apresenta limitações uma vez que possui um objetivo exploratório. Em razão do escopo da pesquisa e espaço disponível outros aspectos que também são relevantes para a avaliação de jogos educacionais como, por exemplo, aspectos pedagógicos e socioculturais não foram contemplados.

Por fim, como estudos futuros se pretende avaliar as heurísticas propostas com um grupo de especialistas para verificar a sua aplicabilidade e também a necessidade ou não de uma ampliação do escopo. Além disso, são planejadas pesquisas junto ao usuário, crianças com discalculia, para analisar a jogabilidade de jogos específicos enquanto intervenções neuropsicológicas, os quais no momento estão sendo adaptados para o contexto brasileiro por meio de uma parceria com pesquisadores da área da psicologia.

\section{Referências}

AGUIAR, M.; BATTAIOLA, A. L. Gameplay: uma definição consensual à luz da literatura. In SBGames, XV Simpósio Brasileiro de Games e Entretenimento Digital. São Paulo, 2016.

BARENDREGT, W. (2006). Evaluating fun and usability in computer games with children. 2006, 189 f. Tese (doutorando em Design) Eindhoven: Technische Universiteit Eindhoven. 2006.

BASTIEN, J. M. C.; SCAPIN, D. L. Ergonomie Criteria for the Evaluation of Human-Computer Interfaces. (Relatório de Pesquisa $N^{\circ}$. 156). INRIA -Institut National de Recherche en Informatique et en Automatique, Rocquencourt, França. 1993.

BASTOS, J. A. et al. The prevalence of developmental dyscalculia in Brazilian public school system. Arquivos de neuro-psiquiatria, v. 74, n. 3, p. 201-206, 2016. 
BIERRE, K. et al. Accessibility in games: Motivations and approaches. White paper, International Game Developers Association (IGDA), 2004.

BUTTERWORTH, B.; VARMA, S., LAURILLARD, D. Dyscalculia: from brain to education. Science, 332(6033), 1049-1053, 2011.

CARVALHO, M. B. et al. An activity theory-based model for serious games analysis and conceptual design. Computers \& Education, 87, 166-181. 2015.

CEZAROTTO, M. A. Recomendações para o design de jogos, enquanto intervenções motivadoras para crianças com discalculia do desenvolvimento. 2016. 188f. Dissertação (Mestrado em Design) Universidade Federal do Paraná, Curitiba. 2016

CEZAROTTO, M. A.; BATTAIOLA, A. L. Game Design Recommendations focusing on children with developmental dyscalculia. In: Zaphiris, Panayiotis, Ioannou, Andri. (Org.). Lecture Notes in Computer Science. 1ed.Switzerland: Springer International Publishing, 2016, v. 9753, p. 463-473. 2016.

CHANDLER, H. M. Manual de produção de jogos digitais. Bookman, 2012. 478 p.

COOPER, C. L. et al. Mental capital and wellbeing. Oxford: Wiley-Blackwell, 2010.

DEHAENE, S. Précis of the number sense. In: Mind \& language, 16(1), pp. 16-36, 2001.

DEHAENE, S.; COHEN, L. Towards an Anatomical and Functional Model of Number Processing In Mathematical Cognition. Butterworth. B. (Orgs.) (Mathematical Cognition - the journal, 1 (1), p. 83120). Psychology Press, 1995.

DESURVIRE, H.; WIBERG, C. Game usability heuristics (PLAY) for evaluating and designing better games: The next iteration. In: International Conference on Online Communities and Social Computing. Springer Berlin Heidelberg, p. 557-566, 2009.

FEDEROFF. M. 2002. Heuristics and usability guidelines for the creation and evaluation of fun in video games. 2002, 52 f. (Mestrado em Ciências). Universidade de Indiana, 2002.

GOMES, A. S.; PADOVANI, S. Usabilidade no ciclo de desenvolvimento de software educativo. In: SBIE Simpósio Brasileiro de Informática na Educação, Juiz de Fora (MG), 2005.

HAASE, V. G. et al. Heterogeneidade Cognitiva nas Dificuldades de Aprendizagem da Matemática: Uma Revisão Bibliográfica. Psicologia em Pesquisa, v. 6, n. 2, p. 139-150, 2012.

HAASE, V. G.; PINHEIRO-CHAGAS, P.; \& ANDRADE, P. M. O. Reabilitação cognitiva e comportamental. In A. L. Teixeira \& A. Kummer (Orgs.) Neuropsiquiatria clinica (pp. 115-123). Rio de Janeiro, RJ: Rubio, 2012.

HUNICKE, R.; LEBLANC, M.; ZUBEK, R. MDA: A formal approach to game design and game research. In: Proceedings of the AAAI Workshop on Challenges in Game AI. p. 1, 2004. 
ISO 9241-210, Ergonomics of human-system interaction - Part 210: Human-centred design for interactive systems, 2010.

JORDAN, P. W. Pleasure with Products: Human Factors for Body, Mind and Soul. In: Human Factors in Product Design: Current Practice and Future Trends, W. Green and P. Jordan (Eds.), Taylor \& Francis UK, 1999.

KADOSH, R. C. et al. Interventions for improving numerical abilities: present and future. Trends in neuroscience and education, v. 2, n. 2, p. 85-93, 2013.

KAPP, K. M. The gamification of learning and instruction: game-based methods and strategies for training and education. John Wiley \& Sons. 2012.

KÄSER, T. et al. Design and evaluation of the computer-based training program Calcularis for enhancing numerical cognition. Frontiers in psychology, v. 4, 2013.

KAUFMANN, L.; VON ASTER, M. The diagnosis and management of dyscalculia. Dtsch Arztebl Int, v. 109, n. 45, p. 767-78, 2012.

KUCIAN, K.; VON ASTER, M. Developmental dyscalculia. European journal of pediatrics, 174(1), $1-13,2015$.

MASLOW, A. Motivation and Personality, Harper and Row, N.Y, 1970.

MATTAR, J. Games em educação: como os nativos digitais aprendem. São Paulo: Person Prentice Hall, 2010. 208 p.

MAYER, R. E. Computer games for learning: An evidence-based approach. MIT Press. 2014.

MIDDLETON, E. L.; SCHWARTZ, M. F. Errorless learning in cognitive rehabilitation: A critical review. Neuropsychological Rehabilitation, v. 22, n. 2, p. 138-168, 2012.

NIELSEN, J. 1995. 10 Usability heuristics for user interface design. Disponível em <http://nngroup. com/articles/ten-usability-heuristics>. Acesso em fevereiro 2017.

PAGULAYAN, R. J. et al. User-centered design in games. In: Handbook for Human-Computer Interaction in Interactive Systems, J. Jacko and A. Sears (Eds.), Lawrence Erlbaum, Mahwah, N.J. pp. 883-906, 2003.

PREECE, J.; ROGERS, Y.; SHARP, H. Design de interação: além da interação homem-computador. Porto Alegre: Bookman, 2005.

RIEBER, P. L. Multimedia Learning in Games, Simulations, and Microworlds. In R. E. Mayer (Ed.), The Cambridge handbook of multimedia learning (1nd ed., pp. 549-567). New York, NY: Cambridge University Press, 2005.

SCHELL, J. A arte de game design: o livro original - The art of game design: a book of lenses. Rio de Janeiro: Elsevier, Campus, 2011. 489p.

SHALEV, R. S.; GROSS-TSUR, V. Developmental dyscalculia. Pediatric neurology, 24(5), 337-342, 2001 
TOBIAS, S. et al. Multimedia learning from computer games. In R. E. Mayer (Ed.), The Cambridge handbook of multimedia learning (2nd ed., pp. 762-784). New York, NY: Cambridge University Press. 2014.

VYGOTSKY, L. S. A formação social da mente: o desenvolvimento dos processos psicológicos superiores. Martins Fontes, 1989.

WILSON, A. J. et al. An open trial assessment of "The Number Race", an adaptive computer game for remediation of dyscalculia. Behavioral and brain functions, 2(1). 2006

WILSON, A. J.; DEHAENE, S. Number sense and developmental dyscalculia. Human behavior, learning, and the developing brain: Atypical development, v. 2, p. 212-237, 2007.

WINN, Brian. The design, play, and experience framework. Handbook of research on effective electronic gaming in education, v. 3, 2008. p. 1010-1024.

\section{Agradecimentos}

Ao programa de bolsas CAPES. 